\title{
MicroRNA-30a-5p promotes proliferation and inhibits apoptosis of human pulmonary artery endothelial cells under hypoxia by targeting YKL-40
}

\author{
HONG TAN*, HUA YAO*, ZHENBANG LIE, GUO CHEN, SHUGUANG LIN and YING ZHANG \\ Department of Cardiology, Guangdong Cardiovascular Institute, Guangdong Provincial People's Hospital, \\ Guangdong Academy of Medical Sciences, Guangzhou, Guangdong 510100, P.R. China
}

Received September 18, 2018; Accepted April 8, 2019

DOI: $10.3892 / \mathrm{mmr} .2019 .10251$

\begin{abstract}
Pulmonary arterial hypertension (PAH) is a fatal and currently incurable cardiopulmonary disease. Numerous microRNAs (miRNAs) serve important roles in the development of PAH. While the expression of miR-30a-5p was downregulated in the lung tissue of rats in a pulmonary hypertension rat model, the expression pattern and function of miR-30a-5p in human PAH remain unclear. Reverse transcription quantitative polymerase chain reaction (RT-qPCR) was used to examine miR-30a-5p and chitinase-3-like protein 1 (YKL-40) mRNA expression levels. The expression levels of YKL-40 and apoptosis-associated proteins were measured by western blot analysis. Cell proliferation assays and flow cytometry analysis were performed to examine cell proliferation and apoptosis, respectively. The association between miR-30a-5p and YKL-40 was determined by a luciferase reporter assay, RT-qPCR and western blot analysis. The relative expression levels of $\mathrm{miR}-30 \mathrm{a}-5 \mathrm{p}$ in plasma were increased in patients with PAH [median=13.23 (25th percentile $=6.388,75$ th percentile $=21.91)]$ compared with normal controls [median $=2.25$ (25th percentile $=1.4,75$ th percentile=3.7). The expression of miR-30a-5p was significantly downregulated while the protein expression of YKL-40 was significantly upregulated in hypoxia-induced human pulmonary artery endothelial cells (HPAECs) when compared with the hypoxia-induced group at $0 \mathrm{~h}$. miR-30a-5p overexpression promoted HPAEC growth and inhibited apoptosis of
\end{abstract}

Correspondence to: Dr Shuguang Lin or Dr Ying Zhang, Department of Cardiology, Guangdong Cardiovascular Institute, Guangdong Provincial People's Hospital, Guangdong Academy of Medical Sciences, 96 Dongchuan Road, Yuexiu, Guangzhou, Guangdong 510100, P.R. China

E-mail: gdsglin@163.com

E-mail: just4zhangying@hotmail.com

*Contributed equally

Key words: pulmonary arterial hypertension, microRNA-30a-5p, chitinase-3-like protein 1 , proliferation, apoptosis
HPAECs under hypoxia. A miR-30a-5p mimic decreased the luciferase activity of a luciferase reporter construct containing YKL-40 3'-untranslated region and also decreased YKL-40 protein expression. YKL-40 overexpression partly alleviated the effects of miR-30a-5p upregulation on proliferation and apoptosis of HPAECs under hypoxia. In conclusion, the data indicated that miR-30a-5p promoted cell growth and inhibited apoptosis of HPAECs under hypoxia by targeting YKL-40. Therefore, the miR-30a-5p/YKL-40 axis may provide a potential target for the development of novel PAH therapies.

\section{Introduction}

Pulmonary arterial hypertension (PAH) is a fatal and currently incurable cardiopulmonary disease (1), characterized by pulmonary vascular remodeling and vasoconstriction that primarily manifests as a progressive elevation in pulmonary artery pressure and vascular resistance (1). Although notable improvements in the therapeutic strategy for PAH have been achieved, the prognosis of PAH remains unsatisfactory (2). In the early pathogenesis of PAH, pulmonary artery endothelial dysfunction serves a vital role; hypoxia is known to target pulmonary artery endothelial cells (PAECs) and exacerbate their dysfunction $(3,4)$. However, the mechanisms by which hypoxia affects PAECs and regulates PAEC proliferation and apoptosis remain unclear.

Accumulating evidence has indicated that microRNAs (miRNAs), a class of evolutionarily conserved small non-coding RNAs, are essential modulators of a variety of genes and cellular processes including cell proliferation and apoptosis (5). A number of aberrantly expressed miRNAs serve a role in the development of PAH (6-9). miR-30a-5p is downregulated in various types of cancer and serves as a tumor suppressor by inhibiting cancer cell proliferation (10). In addition, miR-30a-5p expression was downregulated in the lung tissue of a PAH rat model (8). However, its function in PAH remains unknown.

Chitinase-3-like protein 1 (YKL-40), also known as human cartilage glycoprotein 39 , is a $40 \mathrm{kDa}$ secreted glycoprotein that was first identified in the secretome of a human osteosarcoma cell line (11). Under normal physiological conditions, YKL-40 expression may be observed in different cell types, 
including vascular smooth muscle cells and chondrocytes (12). In cervical cancer, YKL-40 overexpression has been demonstrated to promote cell proliferation (13). In addition, YKL-40 is involved in endothelial dysfunction as a response to endothelial injury (14). As a marker of inflammation and tissue remodeling, elevated YKL-40 has been associated with disease severity and mortality in a number of cardiovascular diseases $(15,16)$. Furthermore, YKL-40 levels are increased in idiopathic PAH (17), but the precise regulatory mechanism of aberrant YKL-40 expression is unknown. We hypothesized that miR-30a-5p may serve an important role in regulating aberrant YKL-40 expression in PAH. In the present study, miR-30a-5p and YKL-40 expression levels were assessed in PAECs in hypoxia and the role of miR-30a-5p overexpression in regulating cell proliferation and apoptosis in PAECs under hypoxic conditions was evaluated. Finally, the regulatory association between miR-30a-5p and YKL-40 was investigated.

\section{Materials and methods}

Clinical samples. A total of 30 patients with PAH (age, $36.70 \pm 10.31$ years; 26 female and four male patients), including those with idiopathic or congenital heart disease- or connective tissue-associated PAH, were enrolled consecutively from January 2016 to March 2018 at Guangdong Provincial People's Hospital (Guangdong, China), none of whom received treatment during their enrollment. A total of 15 healthy normal controls (age, 33.02 \pm 8.52 years; 14 female patients and one male patient) were enrolled during the same time frame for the physical examination portion of the study. All participants involved in this study received oral and written information about the objectives of the study and provided written informed consent, as per the guidelines established in the Declaration of Helsinki. This study was approved by the Research Ethics Committee of Guangdong Provincial People's Hospital, Guangdong Academy of Medical Sciences [approval no. GDREC2017297H(RI)].

Cell culture. Human PAECs (HPAECs) were obtained from ScienCell Research Laboratories, Inc. HPAECs were cultured in endothelial cell medium (ScienCell Research Laboratories, Inc.), consisting of $500 \mathrm{ml}$ basal medium, $25 \mathrm{ml} \mathrm{FBS}$ (cat. no. 0025), $5 \mathrm{ml}$ endothelial cell growth supplement (ECGS; cat. no. 1052). and $5 \mathrm{ml}$ penicillin/streptomycin solution (cat. no. 0503), all purchased from ScienCell Research Laboratories, Inc. The 293T cells were obtained from the Cell Bank of the Chinese Academy of Sciences and maintained in Dulbecco's modified Eagle's medium (Hyclone; GE Healthcare Life Sciences) supplemented with 10\% FBS (Hyclone; GE Healthcare Life Sciences). All cells were maintained at $37^{\circ} \mathrm{C}$ in a humidified incubator under normoxic $\left(21 \% \mathrm{O}_{2}\right.$ and $5 \%$ $\left.\mathrm{CO}_{2}\right)$ or hypoxic $\left(1 \% \mathrm{O}_{2}\right.$ and $\left.5 \% \mathrm{CO}_{2}\right)$ conditions, as described previously $(18,19)$.

Cell transfection and hypoxic treatment. The miR-30a-5p mimic (5'-UGUAAACAUCCUCGACUGGAAG-3') and negative control (NC) miRNA (miR-NC) (5'-UUCUCCGAA CGUGUCACGUTT-3') were purchased from Guangzhou RiboBio Co., Ltd. The full coding sequence of YKL-40 was cloned into pcDNA3.1 plasmids (Invitrogen; Thermo Fisher
Scientific, Inc.) to construct the YKL-40-overexpressing plasmid (pcDNA-YKL-40). The primers used for YKL-40 overexpressing-plasmid construction were as follows: BamHI-forward 5'-cgcggatccgccaccATGGGTGTGAAG GCGTCTCAAAC-3' and XhoI-reverse 5'-ccgctcgagCTA CGTTGCAGCGAGTGCATCCTTGATG-3'. After transfection with $50 \mathrm{nM}$ miR-30a-5p mimic, $50 \mathrm{nM}$ miR-NC or $50 \mathrm{nM}$ miR-30a-5p mimic, plus $0.5 \mu \mathrm{g}$ pcDNA3.1 or $0.5 \mu \mathrm{g}$ pcDNA-YKL-40 plasmid using Lipofectamine ${ }^{\circledR} 2000$ reagent (Invitrogen; Thermo Fisher Scientific, Inc.) for $24 \mathrm{~h}$, HPAECs were exposed to hypoxic conditions $\left(1 \% \mathrm{O}_{2}\right.$ and $\left.5 \% \mathrm{CO}_{2}\right)$ at $37^{\circ} \mathrm{C}$.

RNA extraction and reverse transcription quantitative polymerase chain reaction $(R T-q P C R)$. Total RNA was isolated from HPAECs or plasma using TRIzol ${ }^{\circledR}$ reagent (Thermo Fisher Scientific, Inc.). To analyze miR-30a-5p expression, RT-PCR was performed with specific stem-loop RT primers using the Mir- $\mathrm{X}^{\mathrm{TM}}$ miRNA First Strand Synthesis kit (Takara Biotechnology Co., Ltd) according to the manufacturer's protocol, following which qPCR was performed using the Mir-X ${ }^{\mathrm{TM}}$ miRNA qRT-PCR SYBR ${ }^{\circledR}$ kit (Takara Biotechnology Co., Ltd.). U6 was used as an internal control. To analyze YKL-40 mRNA expression, cDNA was synthesized using the PrimeScript ${ }^{\mathrm{TM}}$ RT reagent kit (Takara Biotechnology Co., Ltd.). YKL-40 mRNA expression was measured using the SYBR ${ }^{\circledR}$ Premix Ex Taq ${ }^{\mathrm{TM}}$ (Takara Biotechnology Co., Ltd.) on an ABI PRISM ${ }^{\circledR} 7500$ real-time PCR system (Applied Biosystems; Thermo Fisher Scientific, Inc.). 18S rRNA served as an internal control. The qPCR conditions were as follows: $95^{\circ} \mathrm{C}$ for $5 \mathrm{~min} ; 40$ cycles at $95^{\circ} \mathrm{C}$ for $15 \mathrm{sec}$ and $60^{\circ} \mathrm{C}$ for $32 \mathrm{sec}$. The relative fold changes in the transcripts were calculated using the $2^{-\Delta \Delta \mathrm{Cq}}$ method (20). The sequences of the primers used were as follows: miR-30a-5p forward, 5'-ACACTCCAG CTGGGTGTAAACATCCTCGACTG-3'; miR-30a-5p reverse, 5'-CTCAACTGGTGTCGTGGA-3'; U6 forward, 5'-CTCGCT TCGGCAGCACA-3'; U6 reverse, 5'-AACGCTTCACGAATT TGCGT-3'; YKL-40 forward, 5'-CTCTACGGCATGCTC AACAC-3'; YKL-40 reverse, 5'-TGGCGGTACTGACTTGA TGA-3'; and 18S rRNA forward, 5'-CCTGGATACCGCAGC TAGGA-3'; 18S rRNA reverse, 5'-GCGGCGCAATACGAA TGCCCC-3'. All reactions were performed in triplicate.

Western blot analysis. Cellular protein was extracted with ice-cold radioimmunoprecipitation assay lysis buffer (Beyotime Institute of Biotechnology) containing a protease inhibitor. The concentration of protein was determined using a BCA protein assay kit (Jiangsu Kaiji Biotechnology Co., Ltd.). A total of $30 \mu \mathrm{g}$ total protein from each sample was separated using 10\% SDS-PAGE and transferred onto a polyvinylidene difluoride membrane. Following blocking with $5 \%$ non-fat milk dissolved in PBS with $0.1 \%$ Tween-20 (PBST) at $25^{\circ} \mathrm{C}$ for $2 \mathrm{~h}$, the membrane was incubated at $4^{\circ} \mathrm{C}$ overnight with the following primary antibodies: Anti-caspase-3 (1:1,000 dilution; cat. no. 9579); anti-caspase-9 (1:1,000 dilution; cat. no. 52873); anti-Bcl-2-associated $X$ protein (Bax; 1:800 dilution; cat. no. 5023); anti-Bcl-2 (1:2,000 dilution; cat. no. 2872); and anti-GAPDH (1:5,000 dilution; cat. no. 5174), all purchased from Cell Signaling Technology, Inc. The membrane was washed with PBST three times and then incubated with the 
horseradish peroxidase-conjugated Rabbit IgG $(\mathrm{H}+\mathrm{L})$ Highly Cross-Adsorbed Secondary antibody (1:20,000 dilution; product no. A16110; Thermo Fisher Scientific, Inc.) at room temperature for $2 \mathrm{~h}$. An enhanced chemiluminescence kit (Thermo Fisher Scientific, Inc.) was then used to perform chemiluminescent detection. The densitometric analysis was performed using Image Pro-Plus 6.0 software (Media Cybernetics, Inc.). The relative protein level was expressed as a relative ratio of the target protein to the reference protein GAPDH. The relative protein level of the target protein of the control group was arbitrarily presented as 1 .

Cell proliferation assay. Cell proliferation was assessed using an MTS assay with a CellTiter $96^{\circledR}$ AQueous One Solution Cell Proliferation Assay kit (Promega Corporation) according to the manufacturer's protocol. HPAECs were cultured in 96-well plates in triplicate at a density of $1 \times 10^{4}$ cells/well prior to transfection, as aforementioned. Following transfection for $24 \mathrm{~h}$, HPAECs were exposed to hypoxic culture conditions using the AnaeroPounch culture system at $37^{\circ} \mathrm{C}$ for $0,24,48$ or 72 h. CellTiter $96^{\circledR}$ AQueous One Solution reagent $(20 \mu \mathrm{l})$ was added to the medium at each time point and incubated for an additional $3 \mathrm{~h}$ at $37^{\circ} \mathrm{C}$ and $5 \% \mathrm{CO}_{2}$ in the dark. The absorbance was read at $490 \mathrm{~nm}$ using a microplate absorbance reader (Multiskan ${ }^{\mathrm{TM}}$ MK3; Thermo Fisher Scientific, Inc.).

Flow cytometry analysis. The cell apoptosis assay was performed using the Annexin V-fluorescein isothiocyanate (FITC) Apoptosis Detection kit (Jiangsu Kaiji Biotechnology Co., Ltd.). Cells $\left(4 \times 10^{5}\right)$ were plated in 6 -well plates. A total of $24 \mathrm{~h}$ after transfection, the cells were exposed to hypoxic conditions using the AnaeroPounch culture system at $37^{\circ} \mathrm{C}$ for $48 \mathrm{~h}$. Then, HPAECs were collected and the cell pellet was re-suspended in $500 \mu \mathrm{l}$ binding buffer. Next, $5 \mu \mathrm{l}$ Annexin V-FITC and $5 \mu 1$ propidium iodide were added to the cell suspension and the cells were cultured in the dark for $15 \mathrm{~min}$ at room temperature. Cell apoptosis was assessed on a flow cytometer (BD Biosciences). Data were acquired using BD FACSDiva software (version 8.0.1; BD Biosciences). Each experiment was repeated three times.

Bioinformatics prediction and luciferase reporter assays. The potential miR-30a-5p binding sites in the YKL-40 3'-untranslated region (UTR) were predicted using TargetScan software human 7.2 (http://www.targetscan.org/). The wild type (wt) YKL-40 3'UTR was amplified and subcloned into the psi-CHECK-2 vector (Promega Corporation), termed the wt-YKL-40 3'-UTR. The wt-YKL-40 3'-UTR was used as a template to create luciferase reporter constructs containing mutated binding sites of miR-30a-5p using the QuikChange ${ }^{\circledast}$ Site-Directed Mutagenesis kit (Stratagene; Agilent Technologies, Inc.), termed the mutant (mut)-YKL-40 3'UTR. The 293T cells were seeded into 24-well plates and co-transfected with $0.5 \mu \mathrm{g}$ wt-YKL-40 3'-UTR plasmid plus $50 \mathrm{nM}$ miR-30a-5p mimics, $0.5 \mu \mathrm{g}$ wt-YKL-40 3'-UTR plasmid plus with $50 \mathrm{nM} \mathrm{NC}, 0.5 \mu \mathrm{g}$ mut-YKL-40 3'-UTR plasmid plus $50 \mathrm{nM}$ miR-30a-5p mimics, or $0.5 \mu \mathrm{g}$ mut-YKL-40 3'-UTR plasmid plus $50 \mathrm{nM} \mathrm{NC}$ using Lipofectamine ${ }^{\circledR} 2000$ (Invitrogen; Thermo Fisher Scientific, Inc.). The cells were then exposed to hypoxic conditions $\left(1 \% \mathrm{O}_{2}\right.$ and $\left.5 \% \mathrm{CO}_{2}\right)$ at

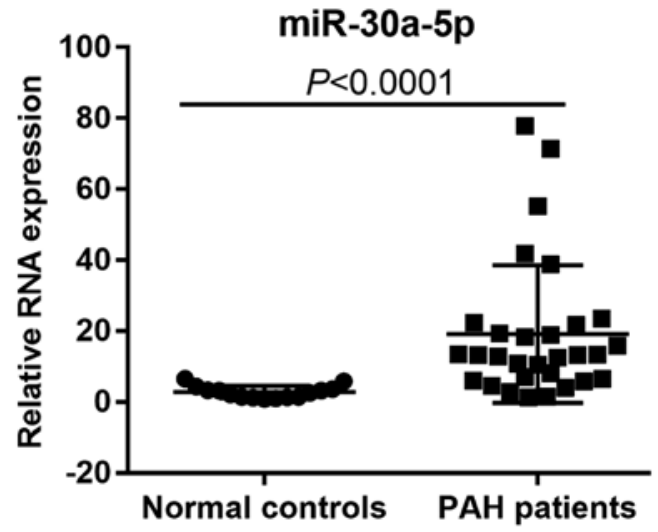

Figure 1. Expression levels of miR-30a-5p in the plasma of normal controls and patients with $\mathrm{PAH}$ detected by reverse transcription quantitative polymerase chain reaction. miR, microRNA; PAH, pulmonary arterial hypertension.

$37^{\circ} \mathrm{C}$ and harvested at $48 \mathrm{~h}$ post-transfection; the luciferase reporter activity was then measured using a Dual-Luciferase ${ }^{\circledR}$ Assay kit (Promega Corporation) following the manufacturer's protocol. Relative luciferase activity was expressed as the ratio of firefly luciferase to Renilla luciferase. Results were obtained from three independent experiments performed in triplicate.

Statistical analysis. The statistical analysis was performed using IBM SPSS Statistics 19.0 software (IBM Corp.). miR-30a-5p expression levels in the plasma of patients with PAH and normal controls were analyzed using the Mann-Whitney $U$ test and described using the median and the 25th and 75th percentiles. All data for experiments in cultured cells are expressed as mean \pm standard deviation. Statistical differences between two groups were evaluated by an unpaired Student's t-test. Statistical differences between multiple groups were evaluated by one-way analysis of variance followed by the Least Significant Difference post-hoc test. $\mathrm{P}<0.05$ was considered to indicate a statistically significant difference.

\section{Results}

miR-30a-5p expression in plasma of patients with PAH. The relative expression levels of miR-30a-5p in plasma were increased in patients with PAH [median $=13.23$ (25th percentile $=6.388,75$ th percentile $=21.91)]$ compared with normal controls [median $=2.25$ (25th percentile $=1.4,75$ th percentile=3.7) $(\mathrm{P}<0.0001$; Fig. 1). The increased level of miR-30a-5p in the plasma of patients with PAH indicated that miR-30a-5p may serve a role in the development and progression of $\mathrm{PAH}$.

Hypoxia decreases miR-30a-5p expression and increases YKL-40 expression in HPAECs. To investigate miR-30a-5p and YKL-40 expression in response to hypoxia, HPAECs were cultured under hypoxic conditions for $0,24,48$ and $72 \mathrm{~h}$. The RT-qPCR results indicated that the relative expression levels of miR-30a-5p under hypoxic conditions for 24, 48 and $72 \mathrm{~h}$ were $0.71 \pm 0.11,0.39 \pm 0.10$ and $0.37 \pm 0.09$, respectively, indicating that hypoxia significantly decreased miR-30a-5p expression in HPAECs (Fig. 2A). Western blot analysis results 


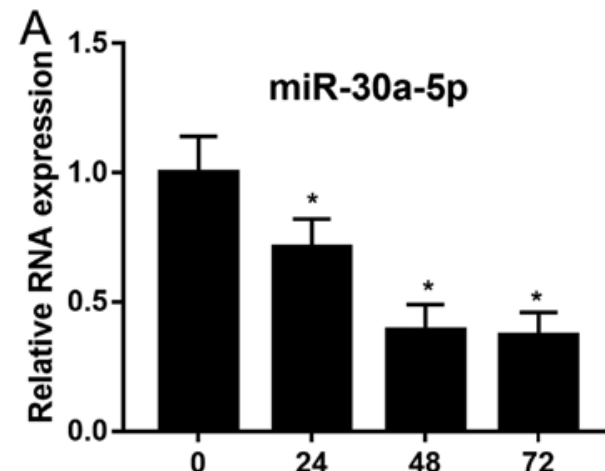

Time after hypoxic treatment (h)
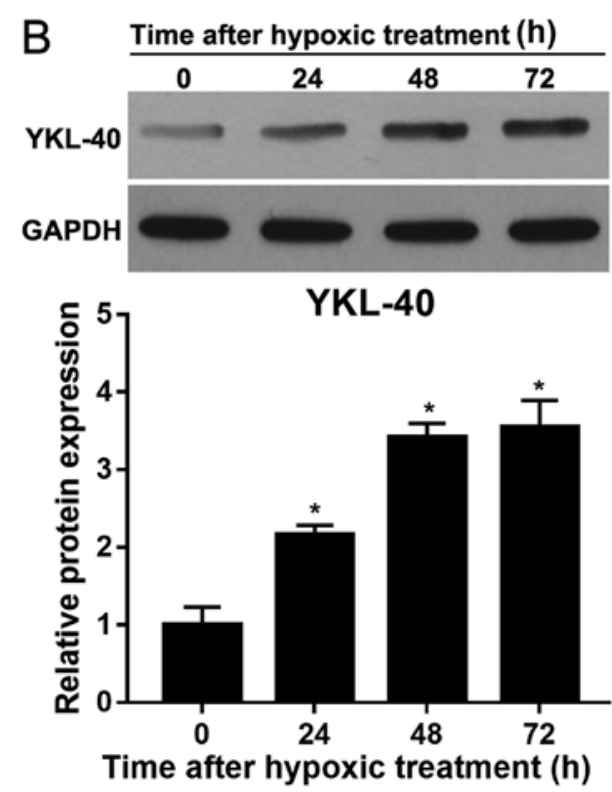

Figure 2. Expression of miR-30a-5p and YKL-40 in HPAECs following hypoxic treatment. (A) The expression of miR-30a-5p at $0,24,48$, and $72 \mathrm{~h}$ after hypoxic treatment, as determined by reverse transcription quantitative polymerase chain reaction. (B) Protein expression levels of YKL-40 at 0,24, 48 and $72 \mathrm{~h}$ after hypoxic treatment, as determined by western blot analysis The upper panel includes a representative western blot analysis gel. The lower panel includes the densitometric analysis of relative YKL-40 expression referenced to GAPDH. ${ }^{\mathrm{P}}<0.05$ vs. $0 \mathrm{~h}$ treated control. miR, microRNA YKL-40, chitinase-3-like protein 1.

demonstrated that the relative protein expression levels of YKL-40 under hypoxic conditions for 24,48 and $72 \mathrm{~h}$ were $2.16 \pm 0.12,3.41 \pm 0.18$ and $3.54 \pm 0.35$, respectively, indicating that hypoxia significantly increased YKL-40 protein expression in HPAECs (Fig. 2B).

miR-30a-5p overexpression promotes proliferation and inhibits apoptosis of HPAECs under hypoxia. In the preliminary experiments, it was identified that the endogenous expression of miR-30a-5p is low in HPA, and the effect of miR-30a-5p inhibitor on miR-30a-5p level was not marked (data not shown). Therefore, only the experiments on miR-30a-5p upregulation were performed. Following transfection with the miR-30a-5p mimic or miR-NC for $24 \mathrm{~h}, \mathrm{HPAECs}$ were exposed to a hypoxic environment for $48 \mathrm{~h}$. The results of the RT-qPCR assay suggested that the expression levels of miR-30a-5p was increased 1987.9-fold in HPAECs transfected with the miR-30a-5p mimic compared with the NC group
(Fig. 3A). miR-30a-5p overexpression significantly promoted the proliferation of HPAECs compared with the miR-NC group under hypoxia for 24, 48 and $72 \mathrm{~h}$ (Fig. 3B). miR-30a-5p overexpression significantly decreased the apoptosis rate in HPAECs compared with the miR-NC group under hypoxia for $48 \mathrm{~h}$ (Fig. 3C). In addition, western blot analysis data demonstrated that miR-30a-5p overexpression decreased the levels of cleaved caspase-3, cleaved caspase- 9 and Bax, while increasing the expression of Bcl-2 in HPAECs under hypoxia for $48 \mathrm{~h}$ (Fig. 3D). These results indicated that miR-30a-5p overexpression promoted proliferation and inhibited apoptosis of HPAECs under hypoxia.

YKL-40 is a target of miR-30a-5p. miR-30a-5p has a potential binding site in the YKL-40 3'-UTR (Fig. 4A). The results of the luciferase reporter assay indicated that luciferase activity was significantly decreased in the 293T cells co-transfected with the wt-YKL-40-3'UTR vector and the miR-30a-5p mimic under hypoxia for $48 \mathrm{~h}$. In contrast, luciferase activity was unaffected in cells co-transfected with the mut-YKL-40-3'UTR vector and miR-30a-5p mimic, relative to the NC group under hypoxia for $48 \mathrm{~h}$ (Fig. 4B). These data indicated that miR-30a-5p directly targeted YKL-40 by binding to its 3'-UTR. The RT-qPCR results demonstrated that miR-30a-5p overexpression had no effect on the mRNA expression levels of YKL-40 in HPAECs under hypoxia for $48 \mathrm{~h}$ (Fig. 4C). However, western blot analysis indicated that the protein expression of YKL-40 decreased $60.3 \%$ in the miR-30a-5p overexpression group compared with the NC group in HPAECs under hypoxia for $48 \mathrm{~h}$ (Fig. 4D). These results indicated that YKL-40 is a target of miR-30a-5p in HPAECs under hypoxia.

Overexpression of $Y K L-40$ partly alleviates the effects of miR-30a-5p upregulation on cell proliferation and apoptosis of HPAECs under hypoxia. To additionally clarify the association between miR-30a-5p and YKL-40 in the regulation of cell proliferation and apoptosis of HPAECs under hypoxia, HPAECs were transfected with $\mathrm{NC}+$ pcDNA, miR-30a-5p + pcDNA, or miR-30a-5p + YKL-40-pcDNA. Following transfection for $24 \mathrm{~h}$, cells were subjected to hypoxia for $48 \mathrm{~h}$. Western blot analysis revealed that the protein expression level of YKL-40 in HPAECs transfected with miR-30a-5p + YKL-40-pcDNA was remarkably upregulated when compared with the cells transfected with miR-30a-5p + pcDNA under hypoxia for $48 \mathrm{~h}$ (Fig. 5A). The results of the MTS assay suggested that cell proliferation was significantly decreased in the miR-30a-5p + YKL-40-pcDNA group compared with that in the miR-30a-5p + pcDNA group, but remained increased compared with that in the $\mathrm{NC}+$ pcDNA group under hypoxia for $48 \mathrm{~h}$ (Fig. 5B). Additionally, apoptosis was significantly upregulated in the miR-30a-5p + YKL-40-pcDNA group compared to the miR-30a-5p + pcDNA group, while it remained decreased compared with that in the $\mathrm{NC}+$ pcDNA group under hypoxia for $48 \mathrm{~h}$ (Fig. 5C). Cleaved caspase-3, cleaved caspase- 9 and Bax expression levels were significantly increased in the miR-30a-5p + YKL-40-pcDNA group compared with the miR-30a-5p + pcDNA group, while the expression of BCL2 was significantly decreased in the miR-30a-5p + YKL-40-pcDNA group compared with the 
A

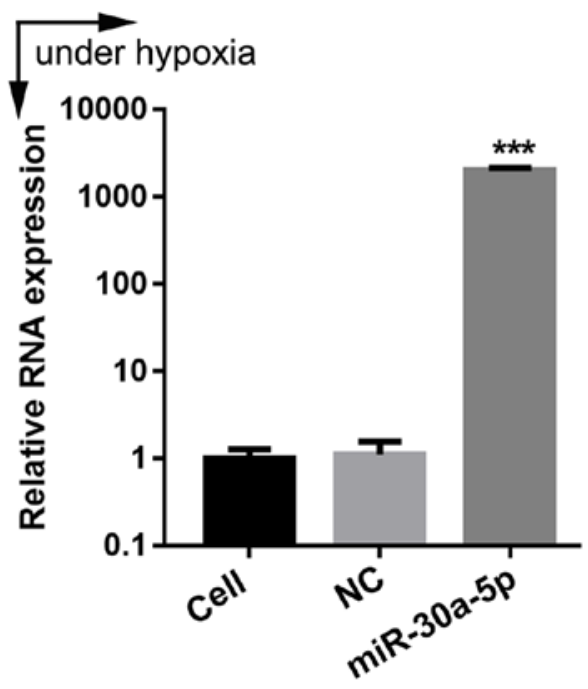

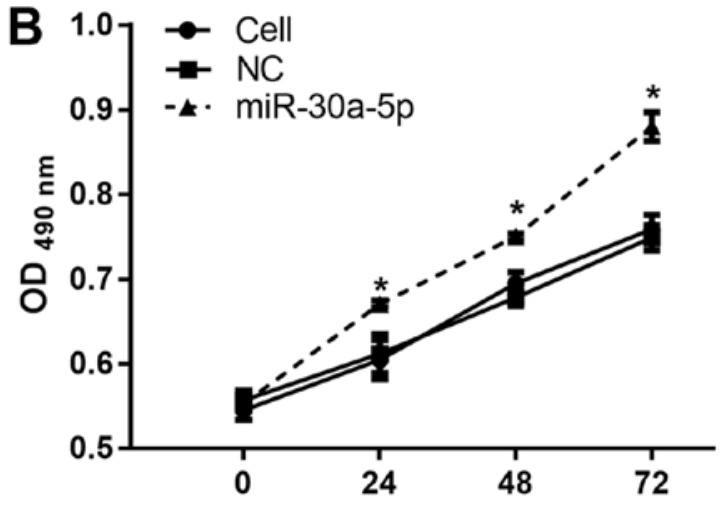

Time after hypoxic treatment (h)

C
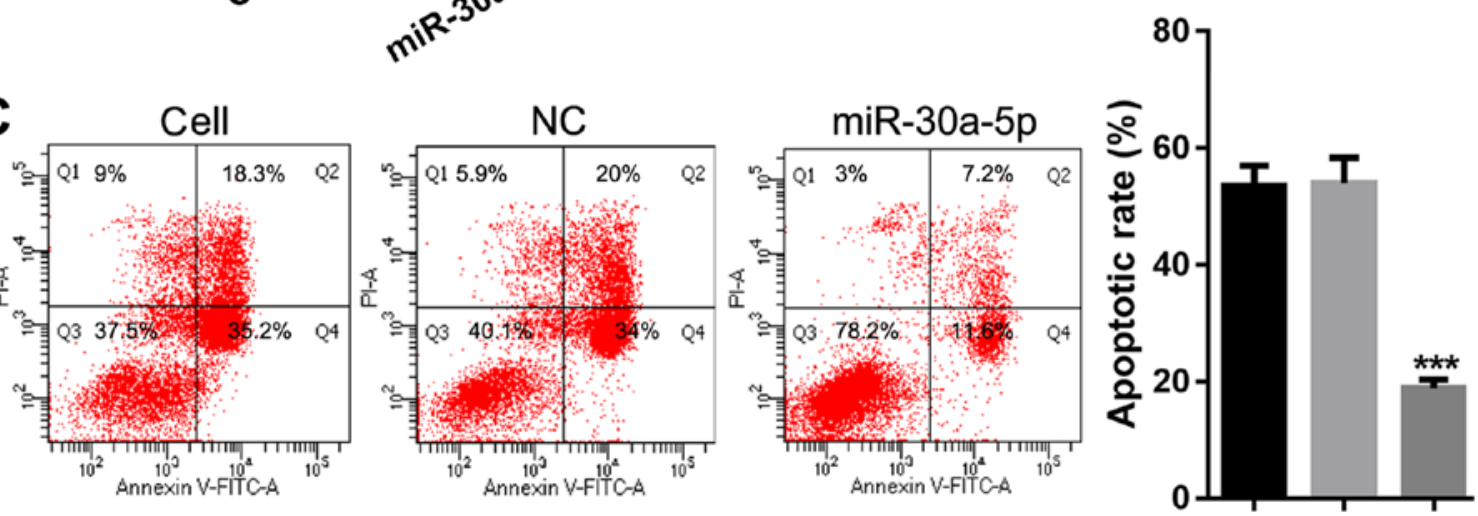

D
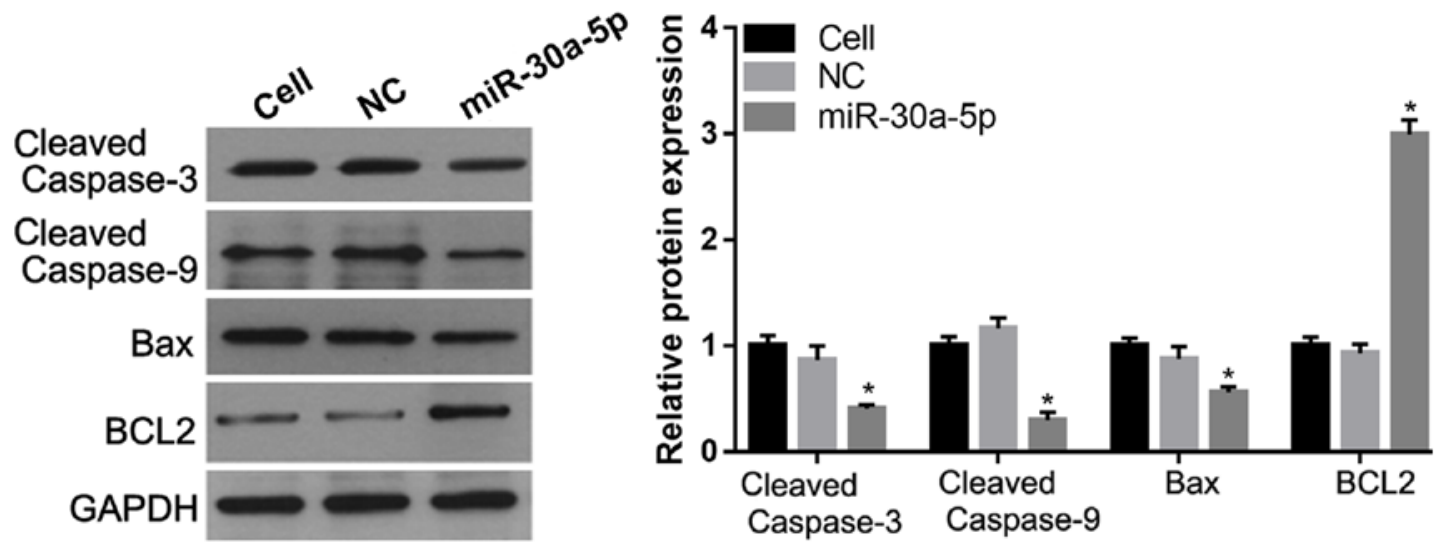

Figure 3. miR-30a-5p overexpression promoted the proliferation and inhibited the apoptosis of HPAECs under hypoxic conditions for 48 h. (A) The level of miR-30a-5p was significantly increased in HPAECs transfected with miR-30a-5p mimics ${ }^{* * *} \mathrm{P}<0.001$ vs. NC. (B) Effect of miR-30a-5p overexpression on the proliferation of HPAECs under hypoxic conditions. ${ }^{*} \mathrm{P}<0.05$ vs. NC. (C) Effect of miR-30a-5p overexpression on the apoptosis of HPAECs under hypoxic conditions. ${ }^{* * *} \mathrm{P}<0.001$ vs. NC. (D) Effect of miR-30a-5p overexpression on the level of cleaved caspase-3, cleaved caspase-9, Bax and Bcl-2 of HPAECs under hypoxia. The left panel includes a representative western blot analysis gel. The lower panel includes the densitometric analysis result of relative protein expression referenced to GAPDH. * $<<0.05$ vs. NC. miR, microRNA; HPAECs, human pulmonary artery endothelial cells; NC, negative control; OD, optical density; Bax, Bcl-2- associated X protein.

miR-30a-5p + pcDNA group under hypoxia for $48 \mathrm{~h}$ (Fig. 5D). These results indicated that the overexpression of YKL-40 partly alleviated the effects of miR-30a-5p upregulation on cell proliferation and apoptosis of HPAECs under hypoxia.

\section{Discussion}

The development of PAH is primarily associated with the aberrant functioning of PAECs, pulmonary artery smooth muscle cells (PASMCs) and vascular fibroblasts (21). Despite the progress in elucidating the function of miRNA in the dysregulated PAECs and PASMCs and in the etiology of PAH, the cellular role and underlying molecular mechanisms remain largely unknown. To the best of our knowledge, the present study is the first to describe the role of miR-30a-5p on cell proliferation and apoptosis in HPAECs under hypoxia. The present study also provided a novel potential therapeutic target for PAH.

Increasing evidence has indicated that miRNAs regulate PAH $(22,23)$. miR-30a-5p is significantly upregulated in colorectal cancer and suppresses proliferation and induces 


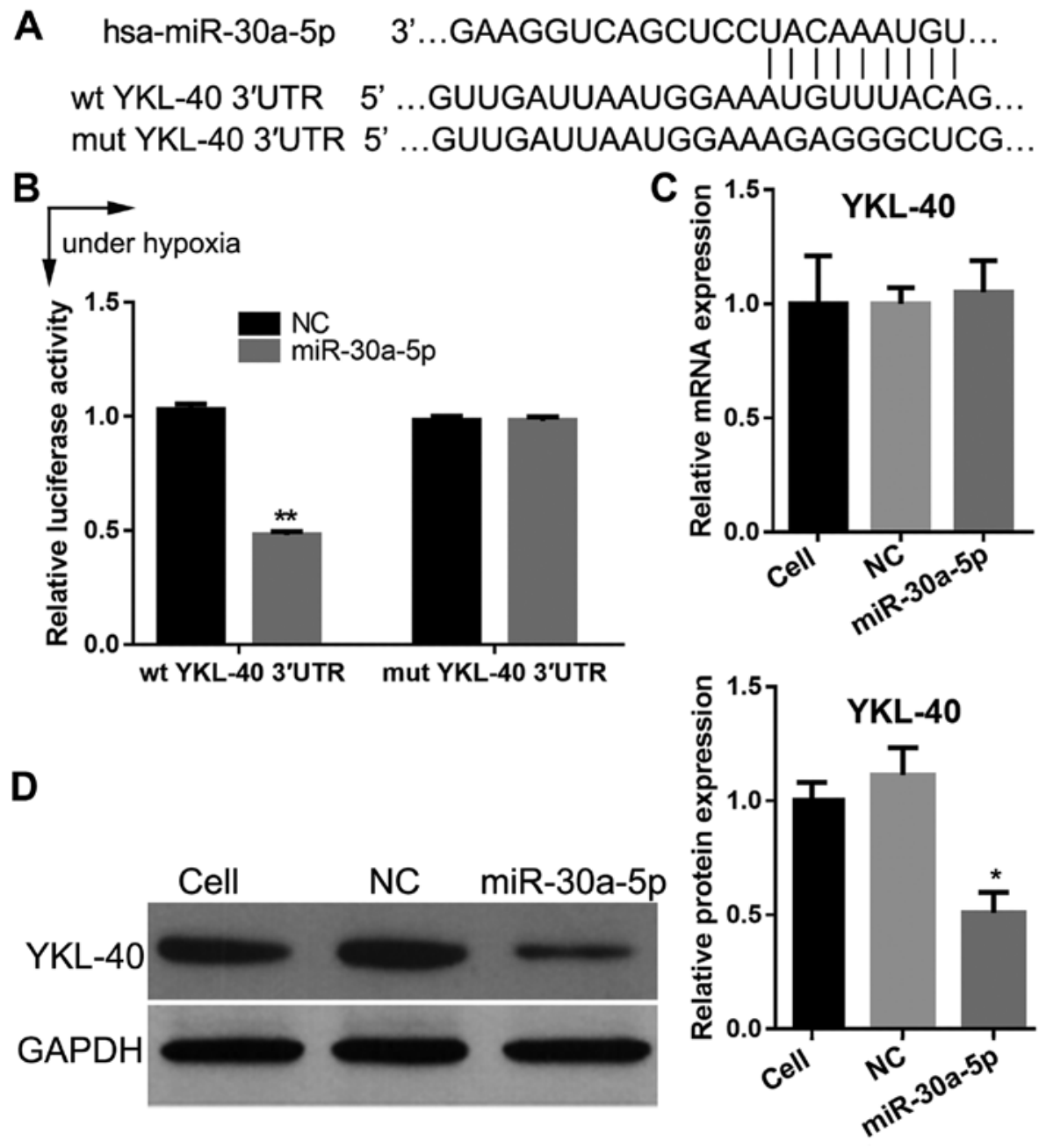

Figure 4. YKL-40 is a direct target of miR-30a-5p. (A) Putative miR-30a-5p binding sequence and corresponding mutant region for YKL-40 mRNA. (B) Effect of miR-30a-5p on the luciferase activity of luciferase reporter constructs containing wt-YKL-40 3'-UTR or mut YKL-40 3'-UTR was measured using a luciferase reporter assay in $293 \mathrm{~T}$ cells under hypoxia for $48 \mathrm{~h}$. ${ }^{* *} \mathrm{P}<0.01 \mathrm{vs}$. NC. (C) Effect of miR-30a-5p overexpression on the mRNA expression of YKL-40 in HPAECs under hypoxia for $48 \mathrm{~h}$. (D) Effect of miR-30a-5p overexpression on the protein expression of YKL- 40 of HPAECs under hypoxia for $48 \mathrm{~h}$. The left panel is a representative western blot analysis gel. The right panel is the densitometric analysis of relative YKL-40 expression referenced to GAPDH. ${ }^{*} \mathrm{P}<0.05$ vs. NC. YKL-40, chitinase-3-like protein 1; miR, microRNA; wt, wild type; mut, mutant type; UTR, untranslated region; NC, negative control; HPAECs, human pulmonary artery endothelial cells.

apoptosis of colon carcinoma cells (24). miR-30a-5p is downregulated in gastric cancer tissues and cell lines and inhibits the proliferation of gastric cancer cells (25). These data suggest that miR-30a-5p is involved in regulating cell proliferation and apoptosis. Although the role of miR-30a-5p has been demonstrated in several cancer types, its role in PAH is not clear. Hypoxia exacerbates pulmonary artery endothelial dysfunction in the early pathogenesis of PAH $(3,4)$; therefore, the expression of miR-30a-5p was examined from 24 to $72 \mathrm{~h}$ in hypoxia-induced HPAECs in the present study. The gradually downregulated expression profile of miR-30a-5p under hypoxic conditions revealed that miR-30a-5p may serve a role in HPAECs. This predication is supported by results indicating that miR-30a-5p overexpression promoted cell proliferation and inhibited apoptosis of HPAECs under hypoxia. Hypoxia-induced HPAEC proliferation and inhibition of HPAEC proliferation is a strategy to attenuate the pathogenesis of PAH $(26,27)$. PAH may also induce apoptosis-resistance in endothelial cells (28). Therefore, we hypothesized that miR-30a-5p may be a suitable therapeutic target for PAH.
miRNAs are able to inhibit the translation or accelerate the degradation of target genes at the 3'-UTR by interacting with the mRNA (29). The identification of target genes is therefore important, in order to illuminate the underlying mechanism of miR-30a-5p in HPAECs under hypoxia. Pulmonary vascular remodeling and inflammation have been implicated in $\mathrm{PAH}$. YKL-40 is considered to serve a role in the process of inflammation and tissue remodeling $(30,31)$. Bioinformatics analysis predicted that miR-30a-5p had a potential binding site in the 3'-UTR of YKL-40. Therefore, YKL-40 may be a potential target of miR-30a-5p. The luciferase reporter assay additionally confirmed that miR-30a-5p directly binds to the 3'-UTR of YKL-40. In addition, it was demonstrated that hypoxia increased YKL-40 expression in HPAECs in the present study. miR-30a-5p overexpression had no effect on the mRNA expression of YKL-40, but it decreased the protein expression of YKL-40 in HPAECs. These data demonstrated that miR-30a-5p may suppress the expression of YKL-40 at the post-transcriptional level and that miR-30a-5p may negatively mediate the protein expression of YKL-40 
A

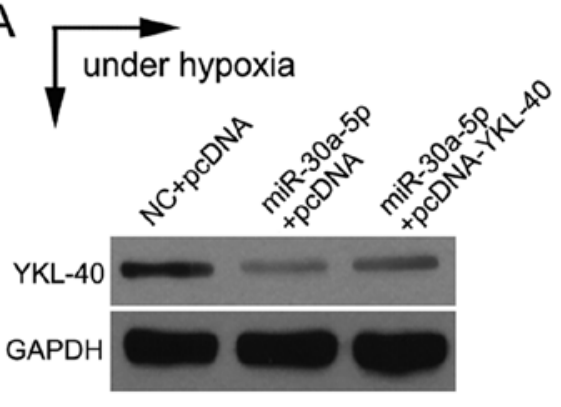

C

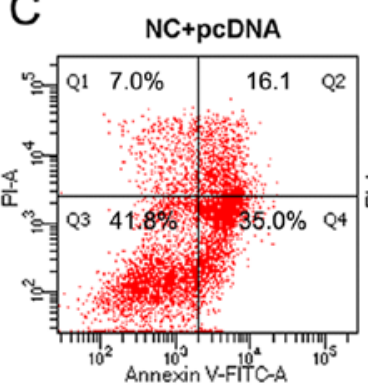

miR-30a-5p+pcDNA

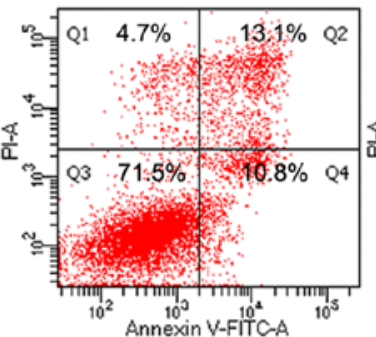

YKL-40

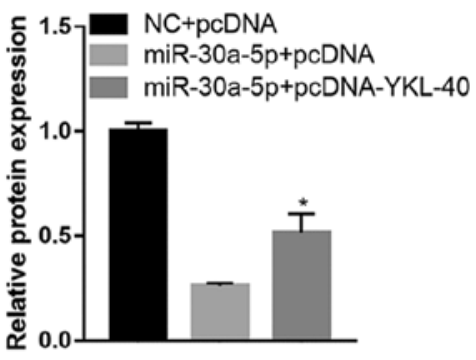

miR-30a-5p+pcDNA-YKL-40

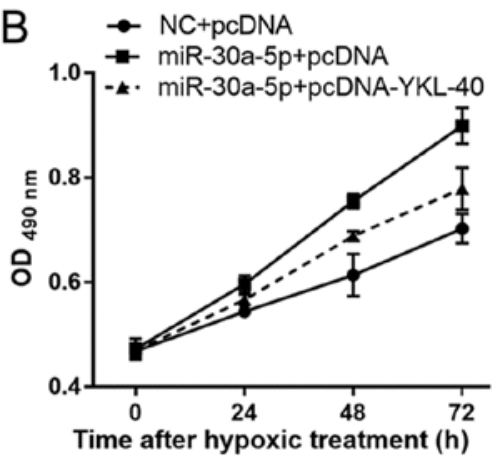

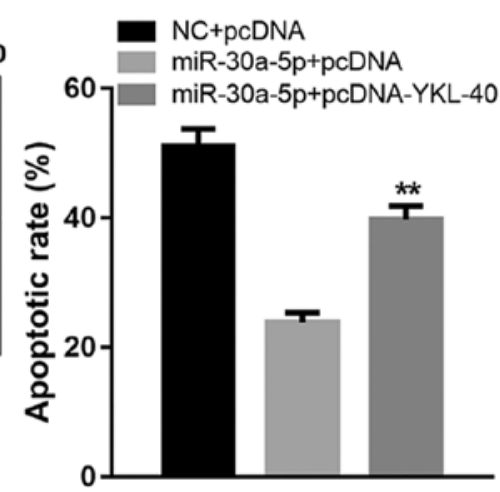
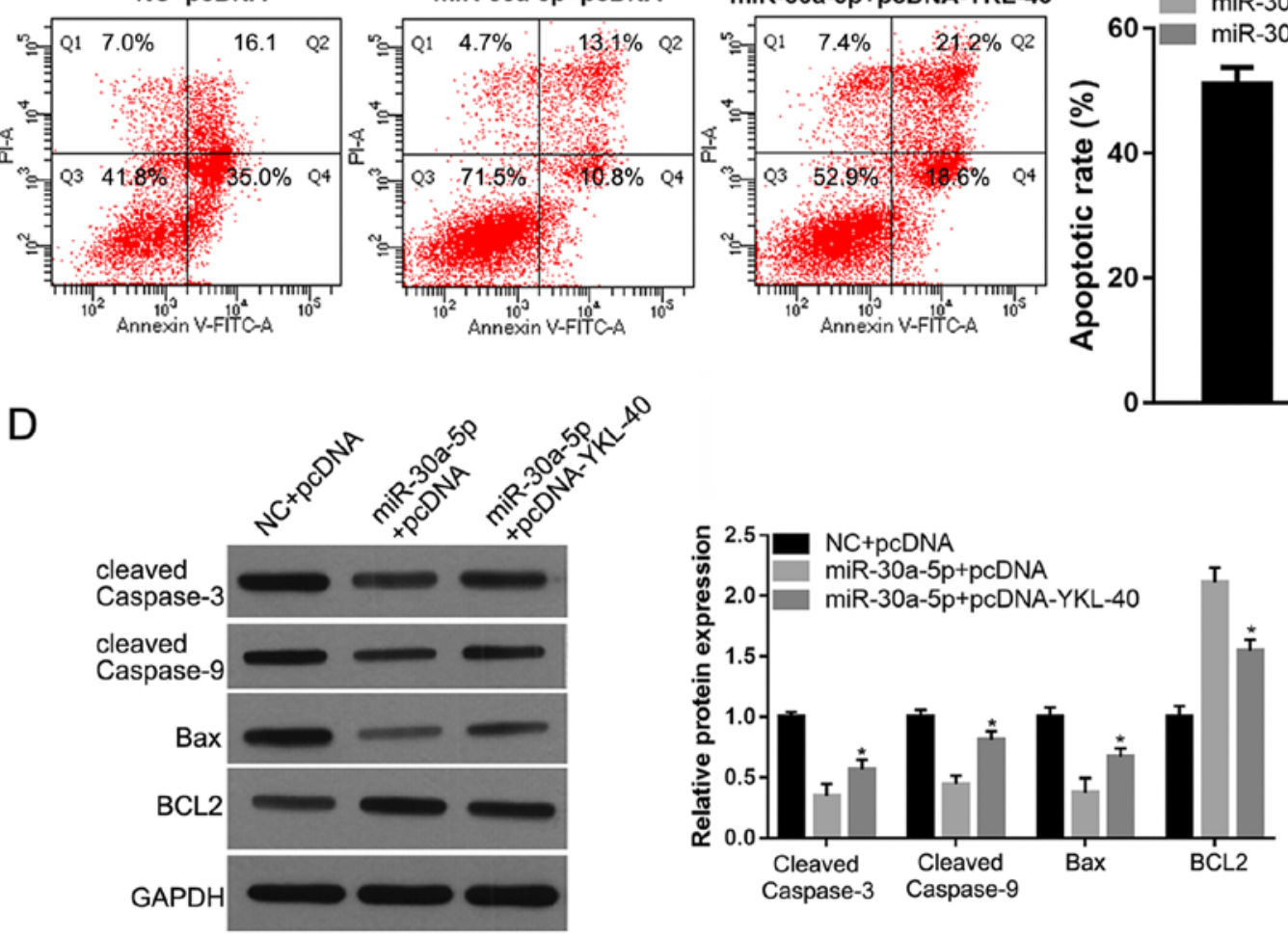

Figure 5. Overexpression of YKL-40 alleviated the effect of miR-30a-5p on cell proliferation and apoptosis in HPAECs under hypoxia for 48 h. HPAECs were pre-transfected with NC + pcDNA, miR-30a-5p + pcDNA, or miR-30a-5p + YKL-40-pcDNA for 24 h and subsequently subjected to hypoxia for 24 h. (A) Western blot analysis was conducted to measure the protein expression of YKL-40. (B) MTS assay was performed to measure the levels of proliferation. (C) Apoptosis assays were performed to measure the apoptotic rate. (D) Western blot analysis was conducted to measure the protein expression of cleaved caspase-3, cleaved caspase-9, Bax and BCL2. ${ }^{*} \mathrm{P}<0.05$ and ${ }^{* *} \mathrm{P}<0.01$ vs. miR-30a-5p + pcDNA. YKL-40, chitinase-3-like protein 1; miR, microRNA; HPAECs, human pulmonary artery endothelial cells; NC, negative control; FITC, fluorescein isothiocyanate; PI, propidium iodide; Bax, Bcl-2-associated X protein; OD, optical density.

in HPAECs. To determine whether YKL-40 serves a pivotal role in miR-30a-5p-induced alterations in cell proliferation and apoptosis, a rescue assay was conducted. It was identified that overexpression of YKL-40 partly alleviated the effects of miR-30a-5p upregulation on cell proliferation and apoptosis of HPAECs under hypoxia. These data suggest that miR-30a-5p may regulate HPAEC proliferation and apoptosis, at least in part, by the direct inhibition of YKL-40 expression. However, the signaling pathway involved in HPAEC proliferation and apoptosis is different. Additional studies are required to illuminate the mechanism of YKL-40 in regulating proliferation and apoptosis in HPAECs under hypoxia.

In HPAECs under hypoxia, the miR-30a-5p expression level was gradually downregulated, while YKL-40 was gradually upregulated, indicating a negative correlation between
YKL-40 and miR-30a-5p. In addition, it was identified that miR-30a-5p expression levels were increased in the plasma of patients with PAH compared with normal controls. Its target YKL-40 also exhibited increased levels in the plasma of patients with PAH compared with that in normal controls (17). This may be explained by the hypothesis that, in order to regulate the abnormally high expression of YKL-40, the human body increases the expression level of its inhibitory factor miR-30a-5p, so as to restrain the excessive expression of YKL-40 to a certain extent. However, this hypothesis requires additional investigation.

There are certain limitations to the present study. Although the effects of miR-30a-5p on cell proliferation and apoptosis of HPAECs under hypoxia were analyzed, direct evidence of the participation of miR-30a-5p in PAH was lacking, requiring 
verification in an animal model. Secondly, the dynamic expression of miR-30a-5p in the plasma of different groups of patients with PAH was not explored. The association between clinical characteristics and the prognosis of PAH requires additional studies. Finally, the effect of YKL-40 knockdown on cell proliferation and apoptosis of HPAECs under hypoxia was not investigated, and additional studies are required to confirm the role of YKL-40 in HPAECs under hypoxia. Despite these limitations, these data indicate the potential role of miR-30a-5p as a novel therapeutic target for PAH.

In summary, miR-30a-5p served pivotal roles in proliferation and apoptosis of HPAECs under hypoxia by targeting YKL-40. The results of the present study provide new insights into the regulatory mechanisms underlying $\mathrm{PAH}$ progression and indicate that the miR-30a-5p/YKL-40 axis may provide a potential target in the treatment of $\mathrm{PAH}$.

\section{Acknowledgements}

Not applicable.

\section{Funding}

The present study was supported by the Scientific Technology Project of Guangdong Province (grant no. 2016A020215227), the Scientific Technology Project of Guangzhou (grant no. 201707010432) and the Natural Science Foundation of Guangdong Province (grant no. 2016A030313798).

\section{Availability of data and materials}

The datasets used and/or analyzed during the present study are available from the corresponding author.

\section{Authors' contributions}

HT was involved in study design and preparation of the manuscript. YZ was involved in data collection and literature analysis. HT and YZ performed the experiments. ZL was involved in data collection, statistical analysis and data interpretation. GC was involved in data collection, and the analyses involving PAH patients and controls. SL contributed to data analysis and interpretation. HY was involved in literature analysis, revising the manuscript and performed the plasma miR-30a-5p expression level experiments. All of the authors read and approved the final manuscript.

\section{Ethics approval and consent to participate}

This study was approved by the Research Ethics Committee of Guangdong Provincial People's Hospital, Guangdong Academy of Medical Sciences [approval no. GDREC2017297H (RI)]. All participants involved in this study received oral and written information about the objectives of the study and provided written informed consent.

\section{Patient consent for publication}

All participants provided written informed consent for the publication of associated data.

\section{Competing interests}

The authors declare that they have no competing interests.

\section{References}

1. Schermuly RT, Ghofrani HA, Wilkins MR and Grimminger F: Mechanisms of disease: Pulmonary arterial hypertension. Nat Rev Cardiol 8: 443-455, 2011.

2. Baptista R, Meireles J, Agapito A, Castro G, da Silva AM, Shiang T, Gonçalves F, Robalo-Martins S, Nunes-Diogo A and Reis A: Pulmonary hypertension in Portugal: First data from a nationwide registry. Biomed Res Int 2013: 489574, 2013.

3. Humbert M, Gerry Coghlan J and Khanna D: Early detection and management of pulmonary arterial hypertension. Eur Respir Rev 21: 306-312, 2012.

4. Helan M, Aravamudan B, Hartman WR, Thompson MA, Johnson BD, Pabelick CM and Prakash YS: BDNF secretion by human pulmonary artery endothelial cells in response to hypoxia. J Mol Cell Cardiol 68: 89-97, 2014.

5. Rupaimoole R and Slack FJ: MicroRNA therapeutics: Towards a new era for the management of cancer and other diseases. Nat Rev Drug Discov 16: 203-222, 2017.

6. Bi R, Bao C, Jiang L, Liu H, Yang Y, Mei J and Ding F: MicroRNA-27b plays a role in pulmonary arterial hypertension by modulating peroxisome proliferator-activated receptor gamma dependent Hsp90-eNOS signaling and nitric oxide production. Biochem Biophys Res Commun 460: 469-475, 2015.

7. Liu Y, Liu G, Zhang H and Wang J: MiRNA-199a-5p influences pulmonary artery hypertension via downregulating Smad3. Biochem Biophys Res Commun 473: 859-866, 2016.

8. Caruso P, MacLean MR, Khanin R, McClure J, Soon E, Southgate M, MacDonald RA, Greig JA, Robertson KE, Masson $\mathrm{R}$, et al: Dynamic changes in lung microRNA profiles during the development of pulmonary hypertension due to chronic hypoxia and monocrotaline. Arterioscler Thromb Vasc Biol 30: 716-723, 2010.

9. Rothman AM, Chico TJ and Lawrie A: MicroRNA in pulmonary vascular disease. Prog Mol Biol Transl Sci 124: 43-63, 2014.

10. Wei W, Yang Y, Cai J, Cui K, Li RX, Wang H, Shang X and Wei D: MiR-30a-5p suppresses tumor metastasis of human colorectal cancer by targeting ITGB3. Cell Physiol Biochem 39: 1165-1176, 2016.

11. Schultz NA and Johansen JS: YKL-40-A protein in the field of translational medicine: A role as a biomarker in cancer patients? Cancers (Basel) 2: 1453-1491, 2010.

12. Pouyafar A, Heydarabad MZ, Mahboob S, Mokhtarzadeh A and Rahbarghazi R: Angiogenic potential of YKL-40 in the dynamics of tumor niche. Biomed Pharmacother 100: 478-485, 2018.

13. Sun L, Wang D, Li H, et al: Significance of high YKL-40 expression regulated by mir-24 in cervical cancer progression and prognosis. Int J Clin Exp Pathol 9: 5128-5137, 2016 (In Chinese).

14. Rathcke CN and Vestergaard H: YKL-40-an emerging biomarker in cardiovascular disease and diabetes. Cardiovasc Diabetol 8: 61, 2009.

15. Kastrup J: Can YKL-40 be a new inflammatory biomarker in cardiovascular disease? Immunobiology 217: 483-491, 2012.

16. Nojgaard C, Host NB, Christensen IJ, Poulsen SH, Egstrup K, Price PA and Johansen JS: Serum levels of YKL-40 increases in patients with acute myocardial infarction. Coron Artery Dis 19: 257-263, 2008.

17. Chen G, Yang T, Gu Q, Ni XH, Zhao ZH, Ye J, Meng XM, Liu ZH, He JG and Xiong CM: Elevated plasma YKL-40 as a prognostic indicator in patients with idiopathic pulmonary arterial hypertension. Respirology 19: 608-615, 2014.

18. Green DE, Murphy TC, Kang BY, Kleinhenz JM, Szyndralewiez C, Page P, Sutliff RL and Hart CM: The Nox4 inhibitor GKT137831 attenuates hypoxia-induced pulmonary vascular cell proliferation. Am J Respir Cell Mol Biol 47: 718-726, 2012.

19. Yuan T, Chen Y, Zhang H, Fang L and Du G: Salvianolic Acid A, a component of salvia miltiorrhiza, attenuates endothelial-mesenchymal transition of HPAECs Induced by Hypoxia. Am J Chin Med 45: 1185-1200, 2017.

20. Livak KJ and Schmittgen TD: Analysis of relative gene expression data using real-time quantitative PCR and the 2(-Delta Delta C(T)) method. Methods 25: 402-408, 2001. 
21. Bienertova-Vasku J, Novak J and Vasku A: MicroRNAs in pulmonary arterial hypertension: Pathogenesis, diagnosis and treatment. J Am Soc Hypertens 9: 221-234, 2015.

22. Nie X, Chen Y, Tan J, Dai Y, Mao W, Qin G, Ye S, Sun J, Yang Z and Chen J: MicroRNA-221-3p promotes pulmonary artery smooth muscle cells proliferation by targeting AXIN2 during pulmonary arterial hypertension. Vascul Pharmacol 116: 24-35, 2019.

23. Courboulin A, Paulin R, Giguere NJ, Saksouk N, Perreault T, Meloche J, Paquet ER, Biardel S, Provencher S, Côté J, et al: Role for miR-204 in human pulmonary arterial hypertension. J Exp Med 208: 535-548, 2011.

24. Baraniskin A, Birkenkamp-Demtroder K, Maghnouj A, Zöllner H, Munding J, Klein-Scory S, Reinacher-Schick A, Schwarte-Waldhoff I, Schmiegel W and Hahn SA: MiR-30a-5p suppresses tumor growth in colon carcinoma by targeting DTL. Carcinogenesis 33: 732-739, 2012

25. Liu Y, Zhou Y, Gong X and Zhang C: MicroRNA-30a-5p inhibits the proliferation and invasion of gastric cancer cells by targeting insulin-like growth factor 1 receptor. Exp Ther Med 14: 173-180, 2017.

26. Chen A, Liu J, Zhu J, Wang X, Xu Z, Cui Z, Yao D, Huang Z, Xu M, Chen M, et al: FGF21 attenuates hypoxiainduced dysfunction and apoptosis in HPAECs through alleviating endoplasmic reticulum stress. Int J Mol Med 42: 1684-1694, 2018.
27. Yu H, Liu J, Dong Y, Xu M, Xu L, Guan H, Xia X and Wang L: Anti-hypoxic effect of dihydroartemisinin on pulmonary artery endothelial cells. Biochem Biophys Res Commun 506: 840-846, 2018.

28. Xu W and Erzurum SC: Endothelial cell energy metabolism, proliferation, and apoptosis in pulmonary hypertension. Compr Physiol 1: 357-372, 2011.

29. Fang F, Chang RM, Yu L, Lei X, Xiao S, Yang H and Yang LY: MicroRNA-188-5p suppresses tumor cell proliferation and metastasis by directly targeting FGF5 in hepatocellular carcinoma. J Hepatol 63: 874-885, 2015.

30. Karalilova R, Kazakova M, Batalov A and Sarafian V: Correlation between protein YKL-40 and ultrasonographic findings in active knee osteoarthritis. Med Ultrason 1: 57-63, 2018.

31. Tong X, Wang D, Liu S, Ma Y,Li Z, Tian P and Fan H: The YKL-40 protein is a potential biomarker for COPD: A meta-analysis and systematic review.Int JChron Obstruct Pulmon Dis 13: 409-418, 2018.

This work is licensed under a Creative Commons Attribution-NonCommercial-NoDerivatives 4.0 International (CC BY-NC-ND 4.0) License. 